\title{
Juvenile Psoriatic Arthritis: A Report from the GRAPPA 2017 Annual Meeting
}

\author{
Devy Zisman, Matthew L. Stoll, Yonatan Butbul Aviel, and Elizabeth D. Mellins
}

\begin{abstract}
Juvenile psoriatic arthritis (JPsA), a subtype of juvenile idiopathic arthritis (JIA), constitutes 5\% of JIA. The literature is inconsistent regarding features of JPsA, and physicians debate whether it is a distinct entity within JIA. A biphasic age of onset distribution has been noted. Early-onset disease is characterized by female predominance, small joint involvement, dactylitis, and positive antinuclear antibodies. Late-onset JPsA resembles adult-onset psoriatic arthritis (PsA), with male predominance, psoriasis, enthesitis, and axial disease. Recent studies report improved outcomes, likely due to the widespread use of traditional and biologic disease-modifying antirheumatic drugs. Conflicting HLA associations have been reported in JPsA, but notably both HLA class I and II allele associations are suggested. Similar to PsA cohorts, subjects with JPsA have a lower frequency of a protective interleukin 23R allele than controls or other JIA subtypes. Data in the Childhood Arthritis and Rheumatology Research Alliance (CARRA) patient registry suggest the aggressive characteristics of JPsA: $24.6 \%$ of children have joint damage 4.6 years after symptom onset. Pediatric and adult PsA classification criteria define different JPsA cohorts within the registry and support a previous suggestion that the International League of Associations for Rheumatology criteria for JPsA may be overly stringent. Increased collaboration between pediatric and adult physicians and comparative research on these clinically related conditions are warranted. (J Rheumatol Suppl. 2018 June;94:11-16; doi:10.3899/jrheum.180131)
\end{abstract}

\section{Key Indexing Terms: \\ JUVENILE IDIOPATHIC ARTHRITIS JUVENILE PSORIATIC ARTHRITIS EPIDEMIOLOGY MANIFESTATIONS CLASSIFICATION CRITERIA PSORIATIC ARTHRITIS}

\section{History of JPsA}

Juvenile psoriatic arthritis (JPsA) is one of 7 subtypes of juvenile idiopathic arthritis (JIA) and constitutes about 5\% of JIA ${ }^{1}$. In 1962, Ansell and Bywaters published the first description of psoriasis in the context of a child with arthritis as part of a case series of what was then called Still disease (now JIA) ${ }^{2}$. In 1976, Lambert, et al published the first case

From the Department of Rheumatology, Carmel Medical Center; The Ruth and Bruce Rappaport Faculty of Medicine, Technion-Israel Institute of Technology; Department of Pediatrics, Ruth Rappaport Children's Hospital, Rambam Medical Center, Haifa, Israel; Department of Pediatrics, Division of Rheumatology, University of Alabama at Birmingham, Birmingham, Alabama; Department of Pediatrics, Divisions of Human Gene Therapy and Allergy, Immunology and Rheumatology, Program in Immunology, Stanford University, Stanford, California, USA.

As part of the supplement series GRAPPA 2017, this report was reviewed internally and approved by the Guest Editors for integrity, accuracy, and consistency with scientific and ethical standards.

D. Zisman, MD, Senior Lecturer, Department of Rheumatology, Carmel Medical Center, and Director of Rheumatology Unit, The Ruth and Bruce Rappaport Faculty of Medicine, Technion-Israel Institute of Technology; M.L. Stoll, MD, PhD, MSCS, Associate Professor, Department of Pediatrics, Division of Rheumatology, University of Alabama at Birmingham; Y. Butbul Aviel, MD, Department of Pediatrics, Ruth Rappaport Children's Hospital, Rambam Medical Center, and The Ruth and Bruce Rappaport Faculty of Medicine, Technion-Israel Institute of Technology; E.D. Mellins, MD, Professor, Department of Pediatrics, Divisions of Human Gene Therapy and Allergy, Immunology and Rheumatology, Program in Immunology, Stanford University.

Address correspondence to Dr. D. Zisman, Department of Rheumatology, Carmel Medical Center, 7 Michal St., Haifa 34362, Israel.

E-mail:devyzisman@gmail.com series of JPsA in a retrospective evaluation of 43 children $^{3}$. An important observation from this study is that arthritis can precede the development of psoriasis by as many as 15 years. These observations were echoed in a case series of 60 children published by Shore and Ansell in 1982, in which the authors also noted features present early in the disease that predicted the subsequent development of arthritis, including nail pits, an asymmetrical arthritis, and a family history of psoriasis ${ }^{4}$. The authors called for the development of criteria that would permit JPsA diagnosis without frank psoriasis. This call was answered by Southwood, et al, who proposed a JPsA definition that required arthritis plus either frank psoriasis or at least 2 minor criteria consisting of nail pits, dactylitis, a psoriatic-like rash, or a family history of psoriasis in a first- or second-degree relative ${ }^{5}$. These criteria, dubbed the Vancouver criteria, were validated in $1996^{6}$. At the time, under both the American College of Rheumatology and European League Against Rheumatology definitions, the juvenile arthritis umbrella was limited to categories currently referred to as oligoarticular, polyarticular, and systemic; psoriatic arthritis (PsA) and spondyloarthritis ( $\mathrm{SpA}$ ) were considered separate diseases ${ }^{7}$. This changed in 1997 when Petty, et al convened the International League of Associations for Rheumatology (ILAR) to generate a set of JIA criteria that included the psoriatic and SpA forms and that generated mutually exclusive categories. The first set of criteria was published in $1998^{8}$, with a second and final version published

Personal non-commercial use only. The Journal of Rheumatology Copyright $\subset$ 2018. All rights reserved. 
in $2004^{9}$. The current diagnostic criteria are summarized in Table 1, although it has been suggested that the ILAR criteria are overly stringent and result in missed JPsA diagnoses ${ }^{10}$.

\section{Clinical Features of JPsA}

Clinically, JPsA is a heterogeneous condition that is generally an asymmetrical arthritis, which frequently extends from oligo- to polyarticular, 6 .,11. Both small and large joint involvement are present in the majority of patients ${ }^{5,12,13}$, with axial disease seen in up to $25 \%{ }^{4}$ and dactylitis in $15-50 \%{ }^{4,5,12}$. Extraarticular features are common. The initial descriptions required psoriasis as an entry criterion, which by definition caused it to be present in $100 \%$ of cases ${ }^{3,4}$. Descriptions published since the Vancouver criteria show psoriasis to be present in $25-60 \%$ of cases, with the remainder of cases diagnosed on the basis of minor criteria $^{5,12,13}$. As noted above, psoriasis develops after the onset of arthritis in about $50 \%$ of cases ${ }^{13}$, underscoring the value of the minor criteria in the diagnosis of this disorder. Additional extraarticular features include nail pits in 50-80\% of cases ${ }^{6,14}$, uveitis in $8-15 \% \%^{3,5,6,14}$, and inflammatory bowel disease (IBD) in at most $1 \%{ }^{15}$. Notably, most descriptions of uveitis in children with JPsA are consistent with the chronic uveitis characteristic of oligo- and polyarticular JIA, rather than the acute anterior uveitis associated with HLA-B27+ $\mathrm{SpA}^{5,6,12}$. Laboratory findings are generally unremarkable, although the antinuclear antibody (ANA) test may be positive in at least $50 \%$ of cases $^{5,6,16}$. Radiographic studies can reveal a variety of abnormalities, including erosive disease, joint space narrowing, and sacroiliitis ${ }^{3,11,17}$. JPsA's outcome has improved considerably over the years following the introduction and widespread use of modern therapeutics. Initial studies revealed functional class III or IV outcomes in nearly one-third of patients ${ }^{6,11}$, while more recently published descriptions have shown remission in over $50 \%$ of sub-

Table 1. Diagnostic criteria for juvenile psoriatic arthritis.

\section{Inclusion criteria}

Arthritis and psoriasis; OR

Arthritis plus at least 2 of the following:

Nail pits or onycholysis

Dactylitis

Psoriasis in a first-degree relative

Exclusion criteria

Arthritis in an HLA-B27-positive male beginning after the sixth birthday AS, ERA, sacroiliitis with IBD, reactive arthritis or acute anterior uveitis, or a history of 1 of these disorders in a first-degree relative

The presence of IgM RF on at least 2 occasions at least 3 months apart

The presence of systemic JIA in a patient

Arthritis fulfilling $\geq$ JIA categories

Criteria are adapted from Petty, et $a l^{9}$. AS: ankylosing spondylitis; ERA: enthesitis-related arthritis; IBD: inflammatory bowel disease; IgM: immunoglobulin M; RF: rheumatoid factor; JIA: juvenile idiopathic arthritis. jects $16,18,19$. There is very little treatment data specifically geared toward JPsA. The German BIKER registry included 127 children with JPsA who were treated with etanercept. This registry reported response rates and a safety profile similar to other JIA categories ${ }^{20}$.

\section{Disagreements about JPsA}

JPsA has provided pediatric rheumatologists with debate topics for many years. The 2 main topics on which there is no consensus are (1) Does JPsA constitute a homogeneous group of patients? and (2) Does JPsA exist as a distinct entity within JIA?

Does JPsA constitute a homogeneous group of patients? Many old and recent studies have shown a biphasic age of onset distribution in children with JPs $\mathrm{A}^{3,5,15,16}$, directing the clinical manifestations. Children with early-onset disease tend to have similar features compared to other children with early-onset JIA, including increased predominance in females, positive ANA, and chronic uveitis; whereas children with onset older in life tend to have features suggestive of SpA, including axial disease, enthesitis, and less small joint disease ${ }^{15,16,21}$. Somewhat unexpectedly, dactylitis appears to be largely associated with early-onset disease, despite its association with adult PsA, as well ${ }^{13,15}$.

Butbul Aviel, et al also showed that JPsA is a heterogeneous condition based upon clinical phenotype. Butbul Aviel, et al retrospectively evaluated 122 patients with JPsA, dividing their cohort into 4 main groups according to clinical manifestations that were comparable to the JIA definition suggested by the ILAR criteria: (1) oligoarticular (55\%); (2) rheumatoid factor (RF)-negative polyarticular (29\%); (3) RF-positive polyarticular (3\%); (4) enthesitis-related arthritis (ERA; $13 \%$ ). Of the 65 patients with oligoarticular onset, $32 \%$ eventually developed polyarticular disease ${ }^{22}$. The study found differences in the course, as well as the outcome, of the disease between the groups. The ERA group tended to be older and to have sacroiliac and hip involvement, whereas the polyarticular group had involvement of small joints of the hand, as well as wrist involvement. They also found that the patients in the polyarticular group had the worst outcomes because they took longer to achieve remission and were more likely to have contractures ${ }^{22}$. Therefore, JPsA is clearly a heterogeneous disorder, resembling in part the heterogeneity that has long been identified in its adult counterpart ${ }^{23}$.

Does JPsA exist as a distinct entity within JIA? Another disputed issue is whether JPsA exists as a distinct entity within JIA. Only a few studies have addressed these uncertainties. Most of the studies have focused on comparing clinical data between groups, but later, new genetics data were published. Stoll, et al compared patients with JPsA and JIA with oligoarticular onset in the first 6 months and found that the JPsA group were older, more likely to have involvement of small joints and wrists, and more likely to extend to a polyarticular course ${ }^{24}$. Even when the comparison

Personal non-commercial use only. The Journal of Rheumatology Copyright @ 2018. All rights reserved. 
was limited to children with age of onset under 5 years, similar clinical differences in joint distribution were observed. These data corroborated the study by Huemer, et $a l$, which showed increased small joint and wrist disease among patients with oligoarticular JPsA as compared to oligoarticular JIA ${ }^{12}$. They were also corroborated by Ravelli, et al, whose findings showed that even ANA+ patients with JPsA tended to be older at disease presentation than the rest of their ANA+ JIA counterparts ${ }^{25}$.

Contradictory data have been published. Butbul Aviel, et al used a case control study to compare 53 children with JPsA to 53 children with JIA: 32 with oligoarticular and 21 with polyarticular onset. Patients were matched by sex, age, date of diagnosis, and articular-onset pattern. There were no differences between the groups in the percentage of patients in the oligoarticular groups who extended to a polyarticular course or in the type of joint involvement. There were limited data on outcome, which suggests that time to first inactive disease with or without treatment was similar in both groups $^{26}$. Therefore, it remains unclear whether there are clinical differences between oligoarticular/polyarticular JIA and oligoarticular/polyarticular JPsA.

Late-onset JPsA, however, appears to be substantially different from ERA. Compared to their counterparts with ERA, children with JPsA are less likely to carry the HLA-B27 marker ${ }^{15,27}$, less likely to have sacroiliitis ${ }^{15,27}$, less likely to have hip involvement ${ }^{16,27}$, and as noted above more likely to have chronic, rather than acute, uveitis. Additionally, psoriatic features such as psoriasis and dactylitis were protective against the development of sacroiliitis ${ }^{19,28}$, whereas hip involvement was a risk factor ${ }^{28}$. Therefore, JPsA is a heterogeneous condition in which children with early-onset are generally similar to children with early-onset JIA, yet children with late-onset disease are clearly distinct from their counterparts with ERA, but do resemble early-onset PsA.

We have likely gleaned as much information as we can from clinical studies. The next step is to use genetics to better delineate the differences between JIA and JPsA, as well as to define correspondences with adult inflammatory arthritides ${ }^{29}$.

\section{Immunogenetics of JPsA}

Analysis of JPsA genetics has the potential to provide clues to the basis of the earlier onset of this disease compared to PsA and to suggest immune mechanisms in JPsA. There are several strategies for identifying JPsA genetic risk factors: testing for associations with HLA alleles, testing candidate genes identified by PsA genome-wide association studies (GWAS), and testing genes that cause monogenic disorders with phenotypic overlap with JPsA.

The first study of HLA association with JPsA, published by Ansell, et al in $1993^{30}$ compared a cohort of 70 children diagnosed with JPsA to 310 controls, all from the United Kingdom. Of the 69 subjects with JPsA, 47 had a strong family history of psoriasis. A statistically significant association with HLA-B27 was observed. In a UK study that examined HLA class II associations across ILAR JIA subtypes, 37 patients with JPsA were analyzed ${ }^{31}$. The DRB $1 * 01 / \mathrm{DQA} 1 * 0101 / \mathrm{DQB} 1 * 0501$ haplotype conferred risk with an OR of 3.8 (95\% CI 1.7-9.8). The most recent study by Hinks, et al used dense single-nucleotide polymorphism (SNP) typing of the HLA region to study 5043 JIA cases, 112 JPsA cases, and 14,390 controls ${ }^{29}$. No JPsA associations reached genome-wide significance $\left(\mathrm{p}<5 \times 10^{-8}\right)$, but the following associations were observed: DRB $1 * 08$ $(\mathrm{p}=0.0003) ;$ DQA $1 * 0401(\mathrm{p}=0.0001) ; \mathrm{DQB} * 1 * 0402$ $(\mathrm{p}=0.0008) ;$ HLA-B27 $(\mathrm{p}=0.003)$; and HLA-C*0602 $(\mathrm{p}=0.008)$. Age-stratified analysis was not reported. These results could reflect 2 subtypes of JPsA or independent genetic associations. Interestingly, the class I associations overlap with PsA associations, and the B27 and C*0602 alleles are associated with different phenotypes in PsA subjects $^{32}$. Overall, to date, HLA association studies in JPsA found varied results, but do suggest both HLA class I and class II associations. Notably, a study of HLA associations in JIA from Murray, et al showed that having more HLA risk alleles predisposed the earlier onset of JIA ${ }^{33}$.

Several mechanisms underlying HLA-B27 associations with rheumatic diseases have been hypothesized. The earliest hypothesis pertained to the ability of HLA alleles to present arthritogenic peptides ${ }^{34}$. However, additional hypotheses have emerged in recent years. One model emphasizes the propensity of HLA-B27 to misfold, causing endoplasmic reticulum stress; another emphasizes the recognition of B27 dimers by KIR3DL2 receptors on CD4+ Th17 cells ${ }^{35}$. For the associated DQ alleles, impaired interactions with accessory molecules that regulate peptide loading of HLA class II molecules have been observed and hypothesized to be a basis for presentation of cryptic epitopes of self-proteins, which break immune tolerance ${ }^{36}$. However, specific, mechanistic contributions of associated HLA class I or class II alleles to JPsA pathogenesis, in particular, have not yet been identified.

Using the approach of testing genetic associations observed for PsA, Hinks, et al studied 1244 JIA cases, 93 JPsA cases, and 5200 controls. The study found a negative association between JPsA and an SNP linked to the minor, protective allele of interleukin 23R (IL-23R), the IL-23 receptor gene ${ }^{37}$. This association was not observed in JIA overall or in other ILAR JIA subtypes. Testing the role of IL-23 in JPsA using pharmacologic blockade of the pathway has not been done to date, and further investigation is warranted.

The approach of testing genes that cause monogenic disorders with some phenotypic overlap with JPsA was used by Day, et $a l^{38}$. Genes causing hereditary periodic fever syndromes were tested in 950 UK white JIA cases, including 67 JPsA cases and 728 controls. The genes tested were $M E F V$

Personal non-commercial use only. The Journal of Rheumatology Copyright @ 2018 . All rights reserved. 
(familial Mediterranean fever), NLRP3 (cryopyrin-associated periodic syndrome), NOD2 (Blau syndrome), and PSTPIP1 [pyogenic arthritis, pyoderma gangrenosum, and acne (PAPA) syndrome]. After correction for multiple testing, 2 SNP were significantly associated with JPsA: rs224204 in $M E F V$ (pyrin), corrected $\mathrm{p}=0.025$, and rs 3806265 in NLRP3 (inflammasome component), corrected $\mathrm{p}=0.04$. These findings raise the possibility of an autoinflammatory basis to JPsA, although these associations have not been reported in PsA GWAS studies or in other JPsA cohorts to date. Another strategy worth considering is DNA sequencing of "JPsA" patients with severe phenotypes; this approach may reveal new monogenic diseases in which the causative gene affects pathways involved in JPsA pathogenesis.

Overall, work to date on JPsA biology, using genetic or other approaches, has been limited by the relative rarity of JPsA and the heterogeneity of JPsA. Collaborative efforts, leveraging the pediatric rheumatology research networks already established in many parts of the world, are needed to make progress in understanding the immunogenetics and immunopathogenesis of JPsA.

\section{Lessons from a Large Database of Children with JPsA}

The CARRA legacy registry contains disease-specific cohorts as defined by treating physicians from over 100 sites in the United States and Canada and collected data on 9450 children with pediatric rheumatic diseases between May 2010 and December 2013. Data from this cohort were retrospectively analyzed in light of the current understanding of PsA reflected in the pediatric and adult rheumatology literature ${ }^{15}$.

The JPsA cohort of 361 children (4.9\%) in this registry is in line with published data on the prevalence of this JIA subtype $^{5,39,40}$. The majority were white $(93.9 \%)$ and non-Hispanic $(91.7 \%)$, with a female predominance $(62 \%)$. Average age at symptom onset was $8.34 \pm 4.57$ years, with a delay of $1.04 \pm 1.46$ years from the first symptom to the first pediatric rheumatology appointment. The most common musculoskeletal manifestation was polyarthritis (55.3\%), followed by oligoarthritis (44.7\%), enthesitis (32.7\%), dactylitis (29.7\%), and sacroiliitis (16.7\%). Dermatologic manifestations included rash in $66.8 \%$ and nail pitting in $37.5 \%$, lower prevalence than those reported for $\mathrm{PsA}^{41}$. A possible explanation is that, in contrast to adult PsA, the articular manifestations precede skin manifestations in $\mathrm{JPsA}^{5,16}$, and disease-modifying antirheumatic drugs (DMARD) may obscure their appearance. The prevalence of ANA $(46.2 \%)$ and RF (4.7\%) positivity in the cohort is in the range reported in other JPsA studies ${ }^{4,5,16,22}$ and in studies of adult PsA ${ }^{42}$. Radiographic evidence of joint damage in $24.6 \%$ of the patients, an average of 4.6 years from the time of symptom onset, despite treatment with conventional and biologic DMARD (52.9\%) is similar to the Southwood, et al findings in $1989^{5}$ before the use of biological therapies. These data were confirmed in a report describing radiographic damage in patients with PsA that underscores the potential of psoriatic joint disease, in general, to cause joint damage ${ }^{43}$ and the importance of early diagnosis and therapy.

Similar to PsA, the JPsA cohort exhibited asymptomatic axial involvement and no correlation between the presence of HLA-B27 and radiographic findings of sacroiliitis or the presence of clinical sacroiliitis ${ }^{4,44}$. At enrollment, half of the patients were treated with glucocorticosteroids $(52.1 \%)$ and biological DMARD (52.9\%), consisting mainly of antitumor necrosis factor- $\alpha$ agents. Conventional DMARD, the most common being methotrexate, were prescribed to $81.4 \%$ of the cohort ${ }^{14}$.

The objective improvement in the clinical variables, including arthritis, psoriasis, nail pitting, dactylitis, and enthesitis, was not accompanied by improvement in scores on health-related questionnaires, highlighting JPsA's potential, as in $\mathrm{PsA}^{45}$, to cause disability beyond the objective measurable variables.

The biphasic age of onset observed in the CARRA cohort, in line with previous JPsA studies ${ }^{4,16}$, may suggest an interesting phenotype of disease presentation according to the age of symptom onset, probably influenced by genetic factors ${ }^{46}$. JPsA manifested in early childhood, predominantly as a disease of females with peripheral arthritis, followed by presentation of late-onset JPsA and early-onset PsA in males with $\mathrm{SpA}$ features, and a later presentation with a tendency to peripheral arthritis.

The recent conception of PsA in adults, in the spectrum of SpA including enthesitis and inflammatory back pain, is the main difference between the adult classification criteria for PsA (CASPAR criteria) ${ }^{47}$ and the ILAR classification criteria ${ }^{9}$ for JIA (Table 2 ). This concept was partially implemented by pediatric rheumatologists in the CARRA registry. In the JPsA cohort, 105 children who did not fulfill the ILAR criteria, but who were diagnosed by their treating physicians, exhibited fewer typical manifestations, such as dactylitis, nail pitting, and psoriasis. On the other hand, 52 additional patients who fulfilled CASPAR criteria for PsA but who were not classified in that way by their pediatric rheumatologists were identified in the entire JIA cohort. Those patients had less psoriasis but more features of SpA, such as enthesitis, inflammatory back pain, IBD, and uveitis. The evidence of asymptomatic enthesitis in patients with JPsA is additional support for this concept ${ }^{48}$.

Awareness of these differences in classification is important to ensure that young adults with JPsA are successfully transferred from pediatric to adult care and that their evaluation and treatment are directed by an appropriate diagnosis, particularly as we learn more about the pathogenesis of JPsA.

To overcome the gaps, increased collaboration between pediatric and adult dermatologists and rheumatologists is needed, such as establishing a study group in the Group for Research and Assessment of Psoriasis and Psoriatic Arthritis

Personal non-commercial use only. The Journal of Rheumatology Copyright $\subset$ $\subset$ 2018. All rights reserved 
Table 2. Comparison between ILAR criteria for JPsA and CASPAR criteria for PsA.

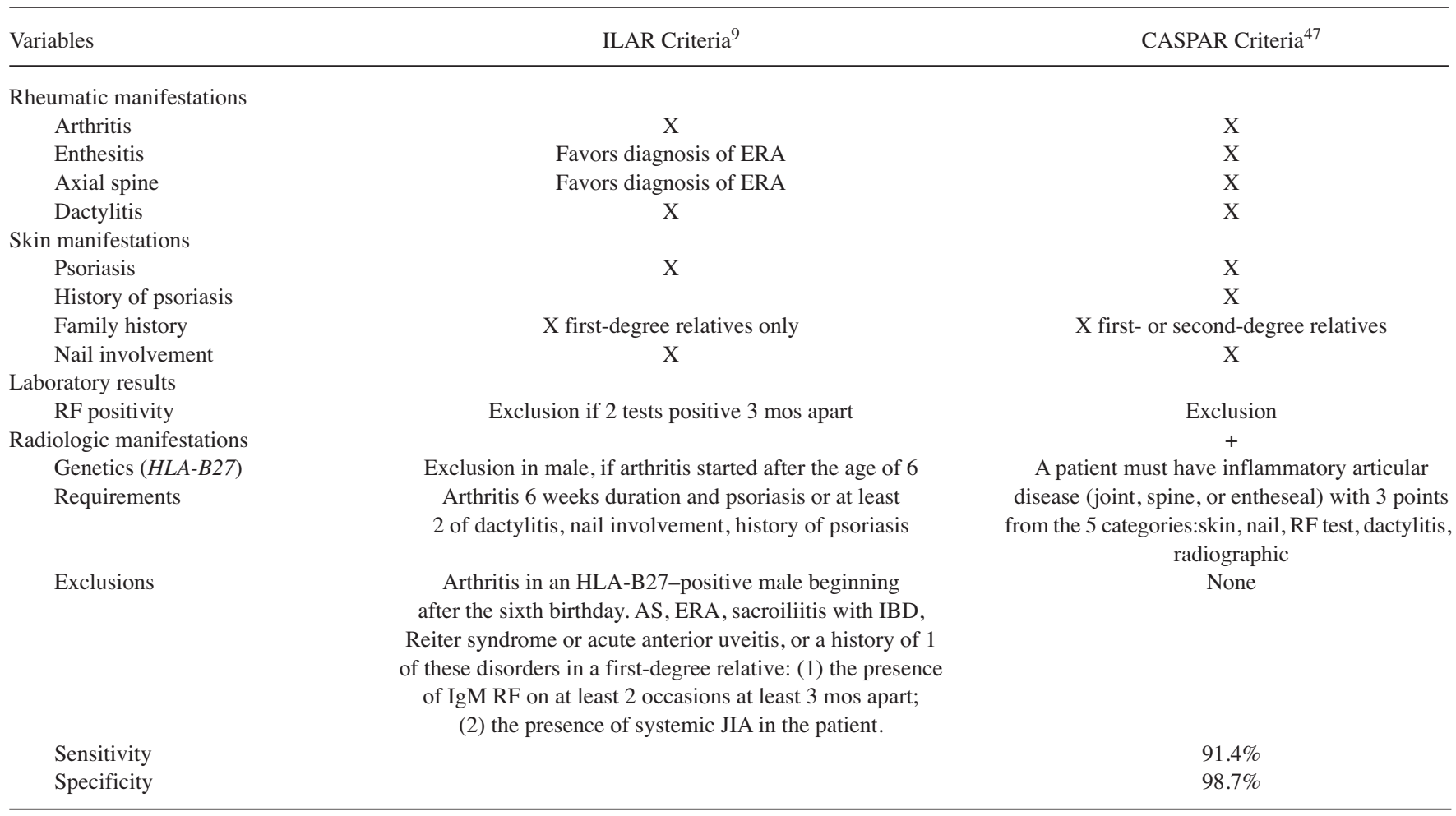

ILAR: International League of Associations for Rheumatology; PsA: psoriatic arthritis; JPsA: juvenile PsA; CASPAR: ClASsification for Psoriatic ARthritis criteria; ERA: enthesitis-related arthritis; RF: rheumatoid factor; AS: ankylosing spondylitis; IBD: inflammatory bowel disease; IgM: immunoglobulin M; JIA: juvenile idiopathic arthritis.

(GRAPPA) organization. This study group may initiate validation of the CASPAR criteria across the age spectrum to reflect the current knowledge.

\section{Patient Transition}

Increased collaboration between pediatric and adult physicians is crucial to the successful transition of young adults with JPsA from pediatric to adult care. Beyond the discussion of clinical aspects, studies on the immunogenicity and pathogenesis of the psoriatic disease in the whole age spectrum may provide the biological knowledge needed to improve diagnosis and therapy.

\section{REFERENCES}

1. Beukelman T, Kimura Y, Ilowite NT, Mieszkalski K, Natter MD, Burrell G, et al. The new Childhood Arthritis and Rheumatology Research Alliance (CARRA) registry: design, rationale, and characteristics of patients enrolled in the first 12 months. Pediatr Rheumatol Online J 2017;15:30.

2. Ansell BM, Bywaters EG. Diagnosis of "probable" Still's disease and its outcome. Ann Rheum Dis 1962;21:253-62.

3. Lambert JR, Ansell BM, Stephenson E, Wright V. Psoriatic arthritis in childhood. Clin Rheum Dis 1976;2:339-52.

4. Shore A, Ansell BM. Juvenile psoriatic arthritis - an analysis of 60 cases. J Pediatr 1982;100:529-35.

5. Southwood TR, Petty RE, Malleson PN, Delgado EA, Hunt DW, Wood B, et al. Psoriatic arthritis in children. Arthritis Rheum 1989;32:1007-13.
6. Roberton DM, Cabral DA, Malleson PN, Petty RE. Juvenile psoriatic arthritis: followup and evaluation of diagnostic criteria. J Rheumatol 1996;23:166-70.

7. Southwood TR, Woo P. Juvenile chronic arthritis. Baillieres Clin Rheumatol 1995;9:331-53.

8. Petty RE, Southwood TR, Baum J, Bhettay E, Glass DN, Manners $\mathrm{P}$, et al. Revision of the proposed classification criteria for juvenile idiopathic arthritis: Durban, 1997. J Rheumatol 1998;25:1991-4.

9. Petty RE, Southwood TR, Manners P, Baum J, Glass DN, Goldenberg J, et al; International League of Associations for Rheumatology. International League of Associations for Rheumatology classification of juvenile idiopathic arthritis: second revision, Edmonton, 2001. J Rheumatol 2004;31:390-2.

10. Stoll ML, Lio P, Sundel RP, Nigrovic PA. Comparison of Vancouver and International League of Associations for rheumatology classification criteria for juvenile psoriatic arthritis. Arthritis Rheum 2008;59:51-8.

11. Wesolowska H. Clinical course of psoriatic arthropathy in children. Mater Med Pol 1985;17:185-7.

12. Huemer C, Malleson PN, Cabral DA, Huemer M, Falger J, Zidek T, et al. Patterns of joint involvement at onset differentiate oligoarticular juvenile psoriatic arthritis from pauciarticular juvenile rheumatoid arthritis. J Rheumatol 2002;29:1531-5.

13. Stoll ML, Nigrovic PA. Subpopulations within juvenile psoriatic arthritis: a review of the literature. Clin Dev Immunol 2006; 13:377-80.

14. Sills EM. Psoriatic arthritis in childhood. Johns Hopkins Med J 1980;146:49-53.

15. Zisman D, Gladman DD, Stoll ML, Strand V, Lavi I, Hsu JJ, et al; CARRA Legacy Registry Investigators. The Juvenile Psoriatic Arthritis Cohort in the CARRA Registry: clinical characteristics, 
classification, and outcomes. J Rheumatol 2017;44:342-51.

16. Stoll ML, Zurakowski D, Nigrovic LE, Nichols DP, Sundel RP, Nigrovic PA. Patients with juvenile psoriatic arthritis comprise two distinct populations. Arthritis Rheum 2006;54:3564-72.

17. Hamilton ML, Gladman DD, Shore A, Laxer RM, Silverman ED. Juvenile psoriatic arthritis and HLA antigens. Ann Rheum Dis 1990;49:694-7.

18. Flatø B, Lien G, Smerdel-Ramoya A, Vinje O. Juvenile psoriatic arthritis: longterm outcome and differentiation from other subtypes of juvenile idiopathic arthritis. J Rheumatol 2009;36:642-50.

19. Ekelund M, Aalto K, Fasth A, Herlin T, Nielsen S, Nordal E, et al; Nordic Study Group of Pediatric Rheumatology (NoSPeR). Psoriasis and associated variables in classification and outcome of juvenile idiopathic arthritis - an eight-year follow-up study. Pediatr Rheumatol Online J 2017;15:13.

20. Windschall D, Muller T, Becker I, Horneff G. Safety and efficacy of etanercept in children with the JIA categories extended oligoarthritis, enthesitis-related arthritis and psoriasis arthritis. Clin Rheumatol 2015;34:61-9.

21. Stoll ML, Punaro M. Psoriatic juvenile idiopathic arthritis: a tale of two subgroups. Curr Opin Rheumatol 2011;23:437-43.

22. Butbul Aviel Y, Tyrrell P, Schneider R, Dhillon S, Feldman BM, Laxer R, et al. Juvenile Psoriatic Arthritis (JPsA): juvenile arthritis with psoriasis? Pediatr Rheumatol Online J 2013;11:11 .

23. Moll JM, Wright V. Psoriatic arthritis. Semin Arthritis Rheum 1973;3:55-78.

24. Stoll ML, Nigrovic PA, Gotte AC, Punaro M. Clinical comparison of early-onset psoriatic and non-psoriatic oligoarticular juvenile idiopathic arthritis. Clin Exp Rheumatol 2011;29:582-8.

25. Ravelli A, Varnier GC, Oliveira S, Castell E, Arguedas O, Magnani A, et al. Antinuclear antibody-positive patients should be grouped as a separate category in the classification of juvenile idiopathic arthritis. Arthritis Rheum 2011;63:267-75.

26. Butbul YA, Tyrrell PN, Schneider R, Dhillon S, Feldman BM, Laxer $\mathrm{RM}$, et al. Comparison of patients with juvenile psoriatic arthritis and nonpsoriatic juvenile idiopathic arthritis: how different are they? J Rheumatol 2009;36:2033-41.

27. Burgos-Vargas R, Vázquez-Mellado J. The early clinical recognition of juvenile-onset ankylosing spondylitis and its differentiation from juvenile rheumatoid arthritis. Arthritis Rheum 1995;38:835-44.

28. Stoll ML, Bhore R, Dempsey-Robertson M, Punaro M. Spondyloarthritis in a pediatric population: risk factors for sacroiliitis. J Rheumatol 2010;37:2402-8.

29. Hinks A, Bowes J, Cobb J, Ainsworth HC, Marion MC, Comeau $\mathrm{ME}$, et al. Fine-mapping the MHC locus in juvenile idiopathic arthritis (JIA) reveals genetic heterogeneity corresponding to distinct adult inflammatory arthritic diseases. Ann Rheum Dis 2017;76:765-72.

30. Ansell B, Beeson M, Hall P, Bedford P, Woo P. HLA and juvenile psoriatic arthritis. Br J Rheumatol 1993;32:836-7.

31. Thomson W, Barrett JH, Donn R, Pepper L, Kennedy LJ, Ollier WE, et al; British Paediatric Rheumatology Study Group. Juvenile idiopathic arthritis classified by the ILAR criteria: HLA associations in UK patients. Rheumatology 2002;41:1183-9.

32. Winchester R, Giles J, Jadon D, Haroon M, McHugh N, FitzGerald O. Implications of the diversity of class I HLA associations in psoriatic arthritis. Clin Immunol 2016;172:29-33.
33. Murray KJ, Moroldo MB, Donnelly P, Prahalad S, Passo MH, Giannini EH, et al. Age-specific effects of juvenile rheumatoid arthritis-associated HLA alleles. Arthritis Rheum 1999;42:1843-53.

34. Benjamin R, Parham P. Guilt by association: HLA-B27 and ankylosing spondylitis. Immunol Today 1990;11:137-42.

35. Powis SJ, Colbert RA. Editorial: HLA-B27: the story continues to unfold. Arthritis Rheumatol 2016;68:1057-9.

36. Busch R, De Riva A, Hadjinicolaou AV, Jiang W, Hou T, Mellins ED. On the perils of poor editing: regulation of peptide loading by HLA-DQ and H2-A molecules associated with celiac disease and type 1 diabetes. Expert Rev Mol Med 2012;14:e15.

37. Hinks A, Martin P, Flynn E, Eyre S, Packham J; Childhood Arthritis Prospective Study-CAPS; BSPAR Study Group, et al. Subtype specific genetic associations for juvenile idiopathic arthritis: ERAP1 with the enthesitis related arthritis subtype and IL23R with juvenile psoriatic arthritis. Arthritis Res Ther 2011;13:R12.

38. Day TG, Ramanan AV, Hinks A, Lamb R, Packham J, Wise C, et al. Autoinflammatory genes and susceptibility to psoriatic juvenile idiopathic arthritis. Arthritis Rheum 2008;58:2142-6.

39. Berntson L, Fasth A, Andersson-Gare B, Kristinsson J, Lahdenne P, Marhaug G, et al; Nordic Study Group. Construct validity of ILAR and EULAR criteria in juvenile idiopathic arthritis: a population based incidence study from the Nordic countries. International League of Associations for Rheumatology. European League Against Rheumatism. J Rheumatol 2001;28:2737-43

40. Hafner R, Michels H. Psoriatic arthritis in children. Curr Opin Rheumatol 1996;8:467-72.

41. Duarte GV, Faillace C, Freire de Carvalho J. Psoriatic arthritis. Best Pract Res Clin Rheumatol 2012;26:147-56.

42. Silvy F, Bertin D, Bardin N, Auger I, Guzian MC, Mattei JP, et al. Antinuclear antibodies in patients with psoriatic arthritis treated or not with biologics. PLoS One 2015;10:e0134218.

43. Kane D, Stafford L, Bresnihan B, FitzGerald O. A prospective, clinical and radiological study of early psoriatic arthritis: an early synovitis clinic experience. Rheumatology 2003;42:1460-8.

44. Williamson L, Dockerty JL, Dalbeth N, McNally E, Ostlere S, Wordsworth BP. Clinical assessment of sacroiliitis and HLA-B27 are poor predictors of sacroiliitis diagnosed by magnetic resonance imaging in psoriatic arthritis. Rheumatology 2004;43:85-8.

45. Taylor WJ. Impact of psoriatic arthritis on the patient: through the lens of the WHO International Classification of Functioning, Health, and Disability. Curr Rheumatol Rep 2012;14:369-74.

46. Queiro R, Tejón P, Coto P, Alonso S, Alperi M, Sarasqueta C, et al. Clinical differences between men and women with psoriatic arthritis: relevance of the analysis of genes and polymorphisms in the major histocompatibility complex region and of the age at onset of psoriasis. Clin Dev Immunol 2013;2013:482691.

47. Taylor W, Gladman D, Helliwell P, Marchesoni A, Mease P, Mielants $\mathrm{H}$, et al; CASPAR Study Group. Classification criteria for psoriatic arthritis: development of new criteria from a large international study. Arthritis Rheum 2006;54:2665-73.

48. Tuttle KS, Vargas SO, Callahan MJ, Bae DS, Nigrovic PA. Enthesitis as a component of dactylitis in psoriatic juvenile idiopathic arthritis: histology of an established clinical entity. Pediatr Rheumatol Online J 2015;13:7. 